\title{
Alterações nasofibrolaringoscópicas da deglutição na encefalopatia crônica não-progressiva
}

\author{
Fiberoptic endoscopic swallowing disorders in chronic encephalopathy
}

\author{
Dayse Manrique ${ }^{1}$, Erich C.M. Melo², Rogério B. Bühler²
}

\section{Resumo}

Objetivo: o objetivo deste estudo é avaliar os resultados da nasofibrolaringoscopia em crianças com encefalopatia crônica nãoprogressiva (ECNP), no diagnóstico de disfagia orofaríngea neurogênica.

Métodos: realizamos a nasofibrolaringoscopia funcional da deglutição em 68 crianças, enquadradas no programa de reabilitação da Associação de Assistência à Criança Deficiente (AACD) - São Paulo, no período de março de 1999 a março de 2000.

Resultados: observamos escape precoce do contraste alimentar para a faringe $(53,0 \%)$, penetração laríngea de contraste alimentar pastoso $(5,9 \%)$, penetração laríngea de contraste alimentar líquido $(4,4 \%)$, aspiração traqueal de contraste alimentar pastoso $(14,7 \%)$, aspiração traqueal de contraste líquido (32,3\%), resíduo do contraste após a deglutição $(7,4 \%)$ e eficácia da tosse com eliminação do contraste das vias aéreas $(44,1 \%)$.

Conclusões: as crianças apresentaram elevados índices de comprometimento da fase faríngea da deglutição. A nasofibrolaringoscopia permitiu o diagnóstico da disfunção orofaríngea nas crianças com ECNP, sendo importante sua realização para indicar a consistência alimentar mais segura, além de identificar a aspiração traqueal, que está relacionada a uma elevada morbidade.

J Pediatr (Rio J) 2002; 78 (1): 67-70: disfagia, endoscopia, encefalopatia crônica.

\section{Introdução}

A deglutição é um processo neuromuscular dinâmico, que compreende, didaticamente, quatro fases: pré-oral e oral (voluntárias); faríngea e esofágica (involuntárias). Os

1. Pós-graduanda (Doutorado) da Disciplina de Otorrinolaringologia e Distúrbios da Comunicação Humana da Univ. Federal de São Paulo-Escola Paulista de Medicina; Médica Assistente do Hospital do Servidor Público Estadual - Francisco Morato de Oliveira - São Paulo; otorrinolaringologista da Clínica de Disfagia da Associação de Assistência à Criança Deficiente (AACD) - São Paulo.

2. Médico Residente do Serviço de Otorrinolaringologia e Cirurgia de Cabeça e Pescoço do Hospital do Servidor Público Estadual-Francisco Morato de Oliveira - SP; otorrinolaringologista voluntário da Clínica de Disfagia da AACD - São Paulo.

Artigo submetido em 08.05.01, aceito em 31.10.01.

\begin{abstract}
Objective: to evaluate the results of fiberoptic endoscopic examination of swallowing safety in children with cerebral encephalopathy in order to identify patients with oropharyngeal dysphagia.

Methods: 68 children from the Association for the Care of Disabled Children (Associação de Assistência à Criança Deficiente -AACD), São Paulo, were submitted to fiberoptic endoscopic examination of swallowing safety from March 1999 to March 2000.

Results: we observed premature spillage (53\%), penetration in a pureed consistency $(5.9 \%)$, penetration in a liquid consistency $(4.4 \%)$, tracheal aspiration in a pureed consistency $(14.7 \%)$, tracheal aspiration in a liquid consistency $(32.3 \%)$, residue $(7.4 \%)$, cough effectiveness $(44.1 \%)$.

Conclusions: children showed a high incidence of pharyngeal swallowing disorders. Tracheal aspiration was the most important disorder because it is related with high morbidity in children with cerebral encephalopathy. The texture and consistency of the patient's diet may be modified to decrease the risk of aspiration and improve swallowing examination.
\end{abstract}

J Pediatr (Rio J) 2002; 78 (1): 67-70: dysphagia, endoscopy, chronic encephalopathy.

distúrbios da deglutição das fases oral e faríngea são muito comuns em crianças com comprometimento neurológico, porém muito pouco estudadas ${ }^{1-5}$.

A disfagia neurogênica compreende as alterações da deglutição, que ocorrem em virtude de uma doença neurológica, com os sintomas e complicações decorrentes do comprometimento sensório-motor dos músculos envolvidos no processo da deglutição ${ }^{6}$. A disfagia neurogênica é particularmente debilitante, com conseqüente desnutrição e problemas pulmonares crônicos, decorrentes da aspiração traqueal. 
Várias afecções neurológicas cursam com disfagia orofaríngea, a qual é subdiagnosticada. As mais comuns são: encefalopatias crônicas, não-progressivas e progressivas, doenças neuromusculares degenerativas, tumores cerebrais e traumatismo craniencefálico.

As manifestações clínicas dos distúrbios da deglutição não são específicas de cada etiologia, mas constituem uma síndrome, que pode cursar com recusa alimentar, fadiga durante a alimentação, escape oral, regurgitação nasal, engasgos, sufocação, asfixia, cianose, tosse durante a alimentação e alteração da qualidade vocal (voz úmida). No entanto, o quadro mais grave é decorrente da aspiração traqueal, que ocorre por inadequação da fase faríngea da deglutição, levando à infecção pulmonar de repetição.

O diagnóstico da disfagia orofaríngea pode ser feito pela correlação dos seus sintomas e sinais com o diagnóstico neurológico previamente estabelecido. Entre os métodos complementares de diagnóstico, destaca-se a videofluoroscopia da deglutição, que é o exame considerado padrão-ouro no diagnóstico das disfagias. A nasofibrolaringoscopia funcional da deglutição tem resultados comparáveis à videofluoroscopia, sendo um método seguro para ser realizado em pacientes de qualquer idade, com a vantagem de um menor custo, não exposição à radiação, além de poder ser realizado à beira do leito. No entanto, não permite o estudo da fase oral e esofágica da deglutição. Outros métodos, como a cintilografia e o salivograma, permitem identificar a aspiração, que é a complicação mais grave dos distúrbios da deglutição.

O objetivo deste estudo é avaliar os resultados da nasofibrolaringoscopia em crianças com encefalopatia crônica não-progressiva (ECNP), no diagnóstico de disfagia orofaríngea neurogênica.

\section{Métodos}

Foi realizado um estudo retrospectivo em 68 crianças com ECNP e quadro clínico de disfagia orofaríngea, no período de março de 1999 a março de 2000, enquadradas no programa de reabilitação da Associação de Assistência à Criança Deficiente (AACD) - São Paulo, sendo seu protocolo de estudo aprovado pelo comitê de ética da UNIFESP - EPM (Universidade Federal de São Paulo - Escola Paulista de Medicina) e da AACD. A nasofibrolaringoscopia funcional da deglutição foi realizada segundo protocolo proposto por Langmore ${ }^{7}$, sendo indicada nas crianças com queixas predominantemente da fase faríngea da deglutição, já que este método não permite o exame da fase oral da deglutição. Os sintomas e sinais relevantes, considerados para o exame complementar, foram: engasgos, sufocação, asfixia, cianose durante alimentação, tosse até dois minutos após alimentação e alteração da qualidade vocal (voz úmida).

O grau de comprometimento motor das crianças avaliadas não era uniforme, mas todas apresentavam importan- tes alterações motoras orais, e sobretudo da fase faríngea da deglutição, além de serem dependentes de cuidador para a alimentação.

As crianças foram avaliadas ambulatorialmente, colocadas na posição sentada e contidas pelo acompanhante para evitar que movimentos espontâneos ou reflexos pudessem interferir com o exame.

O nasofibroscópio que utilizamos foi o Olympus, de $5 \mathrm{~mm}$ de diâmetro, que apresenta deflexão superior e inferior e canal de aspiração. $\mathrm{O}$ equipamento utilizado foi videocâmara Toshiba, videocassete Toshiba, monitor Sony colorido e fitas para videocassete JVC-SX 120.

O fibroscópio foi introduzido preferencialmente na fossa nasal esquerda e progredido pelo assoalho da mesma. Não foi utilizado anestésico nem vasoconstritor tópicos.

Foi realizada, inicialmente, uma avaliação estrutural e funcional, sem utilização do alimento, com a observação das fossas nasais, rinofaringe e esfíncter velofaríngeo durante a deglutição espontânea de saliva. Por deflexão inferior do aparelho, pôde-se observar a base da língua, as valéculas, as paredes laterais e posterior da faringe, os recessos piriformes e a laringe, e a presença de estase salivar nestas estruturas. A mobilidade e a motilidade das pregas vocais foram observadas durante o choro e/ou a deglutição espontânea de saliva, ou durante a fala, quando o paciente apresentava a habilidade de fonação ou de responder a ordens simples. Em seguida, administrou-se o contraste alimentar por via oral.

Os alimentos foram coloridos com anilina (azul ou verde) e foram administrados por um auxiliar, com a utilização de seringa graduada de $10 \mathrm{ml}$. Suco de frutas foi utilizado para a avaliação da consistência líquida (contraste líquido) e iogurte, para a avaliação da consistência pastosa (contraste pastoso).

Foram administrados volumes crescentes de 1,3 e $5 \mathrm{ml}$ de contraste, inicialmente pastoso, e, em seguida, líquido.

Os exames foram integralmente gravados em fitas de videocassete, e posteriormente analisados os seguintes achados:

- escape precoce do contraste para a laringe e/ou recessos piriformes, caracterizado pela presença do alimento na hipofaringe ou na laringe, antes de ser disparado o reflexo da deglutição, que ao exame fibroscópico é identificado pela retroversão epiglótica;

- penetração laríngea, caracterizada pela presença do contraste no vestíbulo laríngeo, que compreende: face laríngea da epiglote, pregas ariepiglóticas, região interaritenóidea, pregas vestibulares e ventrículos, até a face superior das pregas vocais;

- aspiração traqueal, caracterizada pelo fluxo de contraste na região situada inferiormente às pregas vocais, região subglótica e traquéia, em qualquer momento da deglutição; 
- presença de resíduo após a deglutição, caracterizada pelo acúmulo de contraste após a terceira deglutição, na região da base de língua, das valéculas, dos recessos piriformes e da parede posterior da orofaringe;

- presença de tosse eficaz, caracterizada pela capacidade de eliminar o contraste da laringe após a penetração laríngea e/ou aspiração traqueal.

\section{Resultados}

A idade das crianças com ECNP avaliadas variou de 4 meses a 14 anos, com média de 5,3 anos; sendo 40 crianças do sexo masculino $(58,82 \%)$ e 28 do sexo feminino $(41,17 \%)$.

Nas 68 crianças avaliadas, o exame foi alterado em 41 $(60,3 \%)$ dos casos; sendo que alguns pacientes apresentaram mais de um achado alterado ao exame nasofibrolaringoscópico.

Os achados da nasofibrolaringoscopia funcional da deglutição foram: escape precoce do contraste alimentar para a faringe $(53,0 \%)$, penetração laríngea de contraste alimentar pastoso $(5,9 \%)$, penetração laríngea de contraste alimentar líquido (4,4\%), aspiração traqueal de contraste alimentar pastoso $(14,7 \%)$, aspiração traqueal de contraste líquido (32,3\%), resíduo do contraste após a deglutição $(7,4 \%)$ e eficácia da tosse com eliminação do contraste das vias aéreas $(44,1 \%)$. Os resultados estão resumidos na Tabela 1.

No nosso estudo, nenhuma criança apresentou complicações ou intercorrências durante o exame, ou complicação infecciosa decorrente de aspiração após o mesmo.

Tabela 1 - Achados da nasofibrolaringoscopia funcional da deglutição

\begin{tabular}{lcc}
\hline & $\mathbf{N}$ & $\mathbf{\%}$ \\
\hline Escape precoce & 36 & 53,0 \\
Penetração (contraste pastoso) & 4 & 5,9 \\
Penetração (contraste líquido) & 3 & 4,4 \\
Aspiração (contraste pastoso) & 10 & 14,7 \\
Aspiração (contraste líquido) & 22 & 32,3 \\
Resíduo após deglutição & 5 & 7,4
\end{tabular}

\section{Discussão}

O escape precoce do bolo para a faringe foi a alteração mais freqüentemente encontrada, sendo observada em 36 crianças $(53,0 \%)$, em concordância com Langmore e colaboradores ${ }^{8}$. Este achado está relacionado ao comprometimento motor da fase oral da deglutição.
Helfrich-Miller ${ }^{9}$ relata que quanto maior o atraso em disparar o reflexo da deglutição, maior é a chance de aspirar parte do bolo alimentar, porque a via aérea permanece aberta até que o reflexo da deglutição seja disparado. Acreditamos que o escape precoce do bolo para a faringe está relacionada à aspiração traqueal, que acontece antes da deglutição, no momento em que a via aérea encontra-se desprotegida. No presente estudo, não fizemos a caracterização do momento da aspiração em relação à deglutição, mas acreditamos que esta determinação é muito importante para se tentar estabelecer a causa da aspiração traqueal.

No nosso estudo, não observamos ausência do disparo do reflexo da deglutição, mas atraso no disparo, apesar das crianças avaliadas apresentarem grave comprometimento neurológico.

Na nossa avaliação, foi verificada a penetração laríngea de contraste alimentar pastoso em 4 crianças $(5,9 \%)$ e de contraste alimentar líquido em $3(4,4 \%)$.

A aspiração traqueal de contraste pastoso foi observada em 10 crianças (14,7\%), e a aspiração de contraste líquido, em $22(32,3 \%)$. Gisel \& Benson ${ }^{10}$ relataram um índice de $60 \%$ de aspiração somente com líquidos em crianças com paralisia cerebral gravemente comprometidas, e de $26 \%$ em crianças moderadamente comprometidas, à videofluoroscopia. Rogers ${ }^{5}$ relatou, à videofluoroscopia, $38 \%$ de aspiração, sendo que em $96 \%$ destes casos a consistência aspirada foi a líquida. Miller ${ }^{11}$ encontrou $33,3 \%$ de aspiração.

Nesse estudo, a ocorrência de aspiração esteve associada com uma consistência alimentar específica, sendo a consistência líquida mais freqüentemente aspirada $(32,3 \%)$ que a pastosa $(14,7 \%)$, em concordância com Rogers ${ }^{5}$, Casas $^{2}$, Bulchholz $^{6}$, Casas $^{3}$ e Gisel $^{4}$. A aspiração freqüiente de líquido pode ser justificada, pois a viscosidade do bolo altera a deglutição faríngea com relação à duração do contato da base da língua à parede faríngea, a qual é maior para bolos pastosos, de acordo com Lazarus ${ }^{12}$. Kilman ${ }^{13}$ relata que a gravidade pode ajudar no transporte faríngeo de líquido, mas materiais viscosos e sólidos são transportados por atividade muscular. Líquidos tendem a causar mais estase e o fluxo é mais descoordenado, menos coeso, exigindo uma atividade muscular e um controle respiração/ deglutição mais elaborado.

A patogênese da aspiração e penetração laríngea em crianças com ECNP permanece incerta. A aspiração pode acontecer antes, durante e após a deglutição, podendo as causas serem relacionadas a cada um destes momentos. $\mathrm{O}$ escape precoce de contraste para a faringe, a reduzida contração faríngea, as alterações estruturais laríngeas, a coordenação anormal entre motilidade orofaríngea e o fechamento glótico e a presença de resíduo após a deglutição podem ser associados com aspiração, todavia há um grande número de crianças com essas anormalidades que não aspiram. A ocorrência de aspiração pode ser dependente não somente de anormalidades específicas da fase farín- 
gea, como também da coordenação da deglutição com a respiração.

Neste estudo, a presença de resíduo nos recessos piriformes e nas paredes faríngeas, após a deglutição, foi verificada em 5 crianças $(7,4 \%)$. Rogers ${ }^{5}$ encontrou uma frequiência de resíduo de $60 \%$ na avaliação de cinco crianças com paralisia cerebral. Helfrich-Miller ${ }^{9}$ relata que em $100 \%$ dos casos (5 pacientes) há resíduo em valéculas e em recessos piriformes. A estase de resíduos nos recessos piriformes pode resultar de várias razões, como alteração na fase oral com retardo na propulsão do bolo, atraso no disparo do reflexo da deglutição, reduzida elevação laríngea e disfunção do segmento cricofaríngeo. Destes, acreditamos que os mecanismos mais comumente alterados nas crianças com ECNP são as alterações orais motoras e o atraso no disparo do reflexo da deglutição.

Da análise dos resultados, os autores concluíram que as crianças apresentaram elevados índices de comprometimento da fase faríngea da deglutição. A nasofibrolaringoscopia mostrou-se um método seguro de ser realizado em crianças, permitindo o diagnóstico da disfagia orofaríngea. O exame tem importante função no planejamento da alimentação por via oral, com a possibilidade de caracterizar a consistência alimentar mais segura para prevenir a aspiração traqueal. A aspiração foi um achado observado em $32,3 \%$ na consistência líquida e em $14,7 \%$ na pastosa, sendo esta complicação freqüentemente relacionada à elevada morbidade em crianças com ECNP.

\section{Referências bibliográficas}

1. Manrique D. Avaliação da deglutição em crianças com paralisia cerebral tetraespástica: avaliação nasofibrolaringoscópica [tese]. São Paulo: Universidade Federal de São Paulo - Escola Paulista de Medicina; 1998.

2. Casas MJ, Kenny DJ, Mcpherson KA. Swallowing/ventilation interactions during oral swallow in normal children and children with cerebral palsy. Dysphagia 1994; 9:40-6.
3. Casas MJ, Mcpherson KA, Kenny DJ. Durational aspects of oral swallow in neurologically normal children and children with cerebral palsy: an ultrasound investigation. Dysphagia 1995; 10:155-9.

4. Gisel EG, Alphonce E. Classification of eating impairments based on eating efficiency in children with cerebral palsy. Dysphagia 1995; 10: 268-74.

5. Rogers B, Arvedeson J, Buck G, Smart P, Msall M. Characteristics of dysphagia in children with cerebral palsy. Dysphagia 1994; 9: 69-73.

6. Bulchholz DW. Dysphagia associated with neurological disorders. Acta Oto-rhino-laryngologica Belg 1994; 48: 143-55.

7. Langmore SE, Schatz K, Olson N. Fiberoptic endoscopic examination of swallowing safety: a new procedure. Dysphagia 1988; 2: 216-19.

8. Langmore SE, Schatz K, Olson, N. Endoscopic and videofluoroscopic evaluations of swallowing and aspiration. An Otology Rhinology Laryngology 1991; 100: 678-81.

9. Helfrich-Miller KR, Rector KL, Straka JA. Dysphagia: its treatment in the profoundly retarded patient with cerebral palsy. Arch Phys Med Rehabil 1986; 67: 520-25.

10. Gisel EG, Applegate-Ferrante T, Benson J, Bosna JF. Oralmotor skills following sensorimotor therapy in two groups of moderately dysphagic children with cerebral palsy: aspiration vs nonaspiration. Dysphagia 1996; 11:59-71

11. Miller CK, Willging JL, Strife JL, Rudolph CD. Fiberoptic endoscopic examination of swallowing in infants and children with feeding disorders. Dysphagia 1994; 9:266.

12. Lazarus CL, Logmann JA, Radermaker AW, Kahrilas PJ, Pajak T, Lazar R, Halper A. Effects of bolus volume, viscosity, and repeated swallows in nonstroke subjects and stroke patients. Arch Phys Med Rehabil 1993; 74: 1056-70.

13. Kilman WJ, Goyal RK. Disorders of pharyngeal and upper esophageal sphincter motor function. Arch Inter Med 1976; 136: 592-01.

Endereço para correspondência:

Dra. Dayse Manrique

Rua Canário, 112 - apto. 61 - Moema

CEP 04521-005 - São Paulo, SP

Fone: 115542.0645 / 119126.0325

E-mail:daysemanrique@uol.com.br 